\title{
Gender differences on pain threshold, body temperature and reaction time among elderly population after alternate nostril breathing exercises-A comparative study
}

\author{
P. S. L. Saravanan ${ }^{1}$, S. Anu ${ }^{2, *}$, V. Mathava Kumar ${ }^{3}$ \\ ${ }^{1}$ Professor, Institute of Physiology, Madurai Medical College, Madurai, Tamil Nadu, ${ }^{2}$ Professor and Head, ${ }^{3}$ III MBBS, Dept. of \\ Physiology, Velammal Medical College and Hospital, Madurai, Tamil Nadu, India
}

*Corresponding Author: S. Anu

Email: anu.sengottaiyan@gmail.com

Received: $29^{\text {th }}$ August, 2018

Accepted: $19^{\text {th }}$ September, 2018

\begin{abstract}
Introduction: It is a known fact that elderly males have a faster reaction time than females, and females exhibit greater pain sensitivity than males. Results on body core temperature between genders are inconsistent. Alternate Nostril Breathing (ANB) exercises were known to modulate the autonomic nervous system. The present study was done to find whether such differences between elderly males and females on pain sensitivity, body temperature and reaction time still persisted after ANB exercises.

Objectives: 1 . To measure the pain sensitivity, body temperature and reaction time in elderly males and females; 2 : To compare the effects of 30 minutes of ANB exercises on all these parameters between elderly males and females.

Materials and Methods: 30 elderly males $(n=14)$ and females $(n=16)$ with a mean age of 70.3 years participated in the study. Pain sensitivity was measured with digital algometer, temperature with clinical thermometer and reaction time with choice time apparatus before and immediately after ANB exercises.

Results: A significant decrease $(<0.001)$ in all the three parameters was observed after ANB exercises in both genders. No significant difference was observed between elderly males and females on pain sensitivity, body temperature and reaction time to red light, green light \&click sound except for tone $(<0.019)$.

Conclusion: Reaction time became faster; pain sensitivity and temperature decreased immediately after ANB exercise and this could be due to the balancing effect of the autonomic nervous system. No difference existed between genders after ANB except for tone.
\end{abstract}

Keywords: Breathing exercise, Gender, Elderly, Pain, Temperature, Reaction time.

\section{Introduction}

Aging is characterized by gradual deregulation of homeostatic mechanisms in all organ systems and this could be due to ample number of factors including oxidative stress, increasing inflammation and gene variation. ${ }^{1}$ With aging, there is increase in blood pressure, development of arteriosclerosis, increase in blood sugar, altered motility in gastro intestinal tract and bladder, and decrease in lean body mass. ${ }^{2}$ There is increased prevalence of hypertension in India among elderly ranging from $55 \%$ to $72 \%$ in rural and urban areas. ${ }^{3}$ Around $30.4 \%$ of the elderly Indian population also suffers from diabetes mellitus. ${ }^{4}$ Senility is also associated with deterioration of the circadian rhythm resulting in impaired sleep, and altered core body temperature. Impaired sleep is associated with disordered breathing leading to cardiovascular and metabolic complications. Insomnia is associated with increased sympathetic and decreased parasympathetic tone. ${ }^{5}$

Alternate Nostril Breathing (ANB) exercises have a balancing effect on autonomic nervous system. Regular practice of left nostril initiated ANB had shown a decrease in blood pressure and blood sugar indicating an increase in parasympathetic/decrease in sympathetic activity. Studies on the immediate and specific effects of ANB in non-yogic practitioners were inconsistent with varied results. This variation depends on multiple factors like breath rate, nostril with which breathing was initiated and the phase in which it was initiated.

Pain Threshold (PT) is the time at which pain is felt first when a stimulus was given. The stimulus for experimental pain may be of any type like electrical, pressure, thermal, chemical and ischemic pain. Studies had shown that largest changes in values are obtained mainly for pain induced by pressure and electrical stimulation. Women display greater pain sensitivity to all pain modalities than men. ${ }^{6}$ This increased pain sensitivity in women could be due to hormonal factors, endogenous opioid function and genetic factors. ${ }^{7}$ Though estrogen seems to exhibit both pro and antinociceptive effects, nociceptive effect dominates. Estrogen level seems to fluctuate during different phases of menstrual cycle, pregnancy and menopause. ${ }^{8}$ Decreased pain sensitivity in males could be due to the fact that testosterone has more analgesic property and its level almost remains constant without much fluctuation in males. There is increased activation of mu-opioid system in men especially in amygdala and anterior thalamus in response to sustained pain, whereas women exhibited decreased activation of mu-opiod system especially in nucleus accumbens. ${ }^{9}$

Studies on pain perception in old age is inconsistent, with some studies reporting increase in pain threshold with age and few others reporting decrease in pain threshold with age. ${ }^{10}$ But the sex 
differences in perception of pain still continued in old age with women showing greater pain sensitivity than men. Controversial results were observed again with studies on the effect of slow breathing technique on pain threshold. An increase in pain threshold was observed after 6 weeks of slow alternate breathing practice in a study, whereas other studies reported no change in pain threshold immediately after slow breathing as well as after 6 weeks of training. ${ }^{11-13}$

Body temperature is normally kept within control by adjustments in the autonomic nervous system. ${ }^{14}$ As Hypothalamic temperature regulatory mechanisms decompensate in old age, the body temperature of older individuals is less than the normal value of 36.5 degree celcius. ${ }^{15}$ Few studies report no difference in body temperature between elderly males and females, whereas other studies had found an increased mean temperature in elderly females when compared with elderly males. ${ }^{16,17}$ So far, no study was done to find out the effect of breathing practice exclusively on body temperature. This study aims to find out the effect of breathing practice on body temperature in elderly group as well as to find any gender difference in temperature after ANB.

Reaction time is the minimum time taken to respond to a stimulus and it helps in assessing the integrity of the nervous system. Reaction time is faster in males when compared with females of all the age groups. ${ }^{18}$ Slow reaction time in elderly people accounts for falls and injuries. The present study also aims to find out whether such difference between elderly males and females persists after ANB exercise.

The purpose of the present study is to find out gender differences on immediate effects of ANB on pain threshold, body temperature and reaction time in elderly population.

\section{Aim and Objectives}

1. To measure the pain sensitivity, body temperature and reaction time in elderly males and females

2. To study the effect of 30 minutes of ANB exercises on pain sensitivity, body temperature and reaction time in elderly males

3. To study the effect of 30 minutes of ANB exercises on pain sensitivity, body temperature and reaction time in elderly females

4. To compare the effect of 30 minutes of ANB exercises on pain sensitivity, body temperature and reaction time between elderly males and females

\section{Materials and Methods}

The present study was conducted in a private medical college in Madurai over a period of 2 months from June 2017-August 2017. 30 healthy subjects of both the genders aged between 60-90 years were chosen for this study by simple random sampling. The mean age is 70.3 years. They were divided into 2 groups: Group A included 14 elderly males and group B included 16 elderly females. Elderly people who were attending General Medicine O.P were recruited for this study after obtaining their informed written consent and also after ruling out health issues. As we expected ANB to produce at least a 20 point difference in pain sensitivity value between males and females, the present sample size was chosen. This study was done after obtaining clearance from Institutional ethical committee.

Old individuals who had no prior exposure to pranayama and without clinical evidence of any physical illness like diabetes, hypertension, lung diseases and chronic pain syndromes were included in the study. Subjects with ophthalmologic and hearing disorders, with musculo skeletal deformities, suffering from any psychiatric disorder affecting their psychomotor abilities and on pain medication were excluded from the study.

Description of Intervention: Baseline data on VRT\& ART, pain threshold and temperature was measured initially for both Group A \& B. Then both the group participants were taught Alternate nostril breathing exercises to familiarize them with the technique by the author herself, who was a certified yoga instructor graduated under Tamil Nadu Physical Education and Sports University, 2010. ANB involves inhaling through right nostril for a count of 1-5 while the left nostril is occluded \& then exhaling through the left nostril for a count of 1-5 while the right nostril is occluded with no pause in between. Then the same procedure has to be repeated with the left nostril and completing with the right nostril. This is one breathing cycle with duration of 10 seconds. Hence for 1 minute, there will be 6 breathing cycles. Slow breathing is breathing at a rate of around 5-6/min. Once the skill is acquired, in the same individual VRT \& ART, Pain threshold and Temperature was measured immediately after 30 minutes of ANB exercises on the same day without further follow up.

Data Collection Method \& Tools: Baseline data on all participants were collected using structured questionnaire. VRT\& ART was measured with the help of discriminatory and choice reaction time apparatus (Anand Agencies, Pune) between 10am to 12pm every day. VRT was measured for green and red light stimuli and ART was measured for low and high frequency sound stimuli in the sitting posture at around $80 \mathrm{~cm}$ distance. For recording the baseline VRT, initially the subject was instructed to keep pressing the finger on the response button and once he visualizes the stimulus, immediately he has to remove his finger. The response button terminated the clock counter \& the value of VRT was displayed on the screen in milliseconds. This process has to be repeated for 3 times, and the lowest value was taken as the final reaction time. Baseline ART is also recorded in the same way for both for tone \& click sound. After 30 minutes ANB, again VRT \& ART was recorded. 
Pain sensitivity was assessed with the help of a digital Algometer. After switching on the instrument, electrode attached with the algometer is placed on forearm of the subject and the baseline value is kept at 0 . After that the knob was adjusted till the patient perceives pain sensation and then the final displayed reading at which pain is felt were noted. Temperature was measured with the help of oral mercury- in- glass thermometer before and after breathing exercise.

Statistics: The data was entered into MS excel and analysed using SPSS v16.0.VRT, ART, pain threshold and temperature values before and after 30 minutes of ANB were compared using paired $t$ test. Between Gender differences were analysed using paired test. An arbitrary cut off of 0.05 was used to interpret significance of $\mathrm{p}$ value.

\section{Results}

Table 1: Mean age of subjects

\begin{tabular}{|l|c|c|c|}
\hline \multicolumn{2}{|c|}{} & \multicolumn{2}{|c|}{ Age } \\
\cline { 3 - 4 } \multicolumn{2}{|c|}{} & Mean & $\begin{array}{c}\text { Standard } \\
\text { Deviation }\end{array}$ \\
\hline \multirow{2}{*}{ Sex } & Male & 72.9 & 7.0 \\
\cline { 2 - 4 } & Female & 68.1 & 3.2 \\
\hline Total & 70.3 & 5.8 \\
\hline
\end{tabular}

Table 2: ANB induced changes in ART, VRT, pain and temperature before and after 30 minutes of training

\begin{tabular}{|c|c|c|c|c|c|}
\hline & & Mean & $\mathbf{N}$ & Std. Deviation & $\mathbf{P}$ \\
\hline \multirow{2}{*}{$\begin{array}{l}\text { Pair 1 } \\
\text { VRT-Green }\end{array}$} & Before & .23323 & 30 & .040990 & $<0.001$ \\
\hline & After & .22373 & 30 & .044059 & \\
\hline \multirow{2}{*}{$\begin{array}{l}\text { Pair } 2 \\
\text { VRT-Red }\end{array}$} & Before & .24673 & 30 & .046819 & $<0.001$ \\
\hline & After & .23690 & 30 & .047739 & \\
\hline \multirow{2}{*}{$\begin{array}{l}\text { Pair } 3 \\
\text { ART-Tone }\end{array}$} & Before & .23367 & 30 & .040871 & $<0.001$ \\
\hline & After & .22320 & 30 & .046106 & \\
\hline \multirow{2}{*}{$\begin{array}{l}\text { Pair } 4 \\
\text { ART-Click }\end{array}$} & Before & .25470 & 30 & .038726 & $<0.001$ \\
\hline & After & .24380 & 30 & .044179 & \\
\hline \multirow{2}{*}{$\begin{array}{l}\text { Pair } 5 \\
\text { Pain }\end{array}$} & Before & 65.77333 & 30 & 15.015117 & $<0.001$ \\
\hline & After & 60.487 & 30 & 16.4396 & \\
\hline \multirow{2}{*}{$\begin{array}{l}\text { Pair } 6 \\
\text { Temperature }\end{array}$} & Before & 97.43333 & 30 & .840498 & $<0.001$ \\
\hline & After & 97.427 & 30 & .8769 & \\
\hline
\end{tabular}

Table 2 shows a significant difference in all the parameters before and after ANB exercises in elderly population. A significant decrease in reaction time, pain sensitivity and body temperature was observed.

Table 3: Association of gender with 30 minutes of ANB induced differences in ART, VRT, pain and temperature

\begin{tabular}{|l|c|c|c|c|c|}
\hline & Sex & $\mathbf{N}$ & Mean & Std. Deviation & $\mathbf{p}$ \\
\hline \multirow{3}{*}{ Green } & Male & 14 & .0096 & .00970 & 0.941 \\
\cline { 2 - 6 } & Female & 16 & .0094 & .00977 & \\
\hline \multirow{2}{*}{ Red } & Male & 14 & .0083 & .01149 & 0.523 \\
\cline { 2 - 6 } & Female & 16 & .0112 & .01286 & \\
\hline \multirow{2}{*}{ Tone } & Male & 14 & .0039 & .01392 & 0.019 \\
\cline { 2 - 6 } & Female & 16 & .0163 & .01330 & \\
\hline \multirow{3}{*}{ Click } & Male & 14 & .0095 & .01961 & 0.675 \\
\cline { 2 - 6 } & Female & 16 & .0121 & .01424 & \\
\cline { 2 - 6 } & Male & 14 & 5.9500 & 5.56054 & 0.715 \\
\hline \multirow{2}{*}{$\begin{array}{l}\text { Temperat } \\
\text { ure }\end{array}$} & Female & 16 & 4.7063 & 11.45964 & \\
\cline { 2 - 6 } & Male & 14 & -.1571 & .40137 & 0.078 \\
\hline
\end{tabular}

Table 3 shows a significant decrease only in tone of elderly females when compared with males. No significant difference was observed between elderly males and females on pain sensitivity, body temperature and reaction time to red light, green light \&click sound.

\section{Discussion}

The present study shows a significant difference in pain sensitivity, temperature and reaction time before and after ANB exercises according to Table 2. Pain sensitivity had increased significantly ( $p$ value $<0.001$ ) and this could be attributed to increased sympathetic stimulation that occurs with right nostril associated ANB. Improved concentration and attention that occurs 
with slow deep breathing exercises might also contribute to this..$^{19}$ The present study results differ from previous study results showing no change or decreased pain sensitivity after deep breathing exercises. ${ }^{11-13}$

Body temperature decreased significantly ( $\mathrm{p}$ value $<0.001$ ) immediately after 30 minutes of ANB (Table 2). Generally there is decrease in body temperature with advancing age along with loss of diurnal variation in body temperature due to derangement of the temperature regulating mechanisms. ${ }^{20}$ Decrease in body temperature is associated with increased longevity. This decreased temperature noted after ANB could be due to increased skin blood flow as a result of sympathetically mediated vasodilation. In healthy elderly people at rest, there will be a delay in the sympathetically mediated as well as total vasodilation in response to heat along with decreased sympathetic regulation. ${ }^{21}$ Breathing exercises were shown to correct this defect by modifying the autonomic activity. As aging is associated with decrease in the number and myelination of Ay fibres, this increased pain sensitivity would help them to be aware of injuries immediately so that proper measures could be taken.

According to Table 2, reaction time was found to decrease significantly for green light ( $p$ value $<0.001)$, red light ( $\mathrm{p}$ value $<0.001$ ), tone ( $\mathrm{p}$ value $<0.001$ ) and click ( $\mathrm{p}$ value $<0.001$ ) after ANB. The faster reaction time after ANB could be due to increased sympathetic activity and ascending reticular activity. The results of our study is in accordance with a study done on 16 adults where right nostril initiated ANB decreased reaction time and left nostril initiated ANB increased reaction time. ${ }^{22}$

There are two techniques of ANB. One is left nostril initiated ANB and another one is right nostril initiated ANB. Left nostril initiated ANB was shown to increase parasympathetic activity and right nostril initiated ANB was shown to increase sympathetic activity. In the present study, as breathing activity was initiated in the right nostril in inhalation and not in the left nostril, the increase in pain sensitivity, decrease in body temperature and reaction time might be due to increased sympathetic activity.

There was no significant difference in pain sensitivity, body temperature and reaction time for red light, green light and click between elderly males and females after ANB. This shows the modulating effect breathing exercises on autonomic nervous system. A significant decrease ( $p$ value $<0.019$ ) was observed for tone in females when compared to males after ANB. This differs from a study done on young medical students where reaction time for tone and click remained the same for both males and females. The faster reaction time for tone could be due to the fact that auditory stimulus takes less time to reach the cortex than visual stimulus and this is in agreement with the results of a study in elderly Indian population which showed faster auditory reaction time in females when compared to males. ${ }^{23,24}$

\section{Limitation}

Study group was small and the response obtained was of short term. Long term study has to be done to obtain consistent results. As pain sensitivity varies with the type of experimental stimuli and the present study was done only with electrical stimulus, future studies should elicit pain sensation with other type of stimuli too.

\section{Conclusion}

Right nostril initiated ANB increased pain sensitivity, decreased body temperature and reaction time for light and sound in elderly males and females. This confirms the increased activity of sympathetic nervous system with this type of breathing. ANB can be practiced regularly to increase their alertness and concentration and to prolong their life span. There was no difference in pain sensitivity, body temperature and reaction time for red light, green light and click between elderly males and females. There is decrease in tone in females when compared to males.

\section{Acknowledgement}

The authors would like to acknowledge Dr. K. Jayashree, Assistant professor, Department of Community Medicine, Velammal Medical College for her help in statistical analysis and Ms. M. Dharani Devi, Technician, Department of Physiology, Velammal Medical College for her technical support.

\section{Conflict of Interest: None}

\section{References}

1. Jeremy Walston, Evan C. Hadley, Luigi Ferrucci, Jack M.G uralnik, Anne B Newman, Stephanie A. Studenski et al. "Research Agenda for Frailty in Older Adults: Toward a Better Understanding of Physiology and Etiology: Summary from the American Geriatrics Society/National Institute on Aging Research Conference on Frailty in Older Adults". J Am Geriatr Soc. 2006;54:991-1001.

2. Boss GR, Seegmiller JE. “Age related Physiological Changes and their clinical significance, In Geriatric Medicine". West J Med. 1981;135(6):434-440.

3. Hypertension Study Group. "Prevalance, awareness, treatment and control of hypertension among the elderly in Bangladesh and India: a multicentric study". Bull World Health Organ. 2001;79:490-500.

4. Jain A, Paranjape S. "Prevalence of type 2 diabetes mellitus in elderly in a primary care facility". Indian $J$ Endocrinol Metab. 2013;17:318-22.

5. Cecilia Castro-Diehl, Ana V. Diez Roux, Steven Shea. "Sleep Duration and Quality in Relation to Autonomic Nervous System Measures: The Multi- Ethnic Study of Atherosclerosis". Sleep. 2016;39(11):1927-1940.

6. Riley JL, Robinson ME, Wise EA, Myers CD, and Fillingim RB. "Sex differences in the perception of noxious experimental stimuli: A meta-analysis". Pain. 1998;74:181-187. 
7. Bartley E.J and Fillingim R.B "Sex differences in pain: a brief review of clinical and experimental findings". $\mathrm{Br} J$ Anaesth. 2013:111(1):52-58.

8. Craft R M. "Modulation of pain by estrogens". Pain. 2007;132:S3-S12.

9. Zubieta JK, Smith YR, Bueller JA, Xu Y, Kilbourn MR, Jewett DM et al. "Mu-opiod receptor-mediated antinociceptive responses differ in men and women". $J$ Neurosci. 2002;22(12):5100-7.

10. Hanan G. Eltumi, Osama A. "Tashani. Effect of Age, Sex and Gender on Pain Sensitivity: A Narrative Review". The Open Pain Journal. 2018;11;ISSN 1876-3863.

11. Busch V, Mageri W, Kern U, Haas J, Hajak G, Eichhammer P. "The Effect of Deep and Slow Breathing on Pain Perception, Autonomic Activity, and Mood Processing-An Experimental Study". Pain Medicine. 2012;13(2):215-228.

12. Arinola O Sanya, Ademola M. Adebiyi. "Effect of Controlled Breathing on Pain Tolerance". Afr J Biomed Res. 2000;3:81-84.

13. Larsen, Kalee L. "The effect of slow deep breathing on pain-related variables in osteoarthritis". WWU Graduate School Collection. 2015; 447. https://.wwu.edu/wwuet/447

14. Jody L. Greaney, W Larry Kenney and Lacy M. Alexander. "Sympathetic regulation during thermal stress in human aging and disease". Auton Neurosci. 2016;196:81-90.

15. Gunes UY, Zaybak A. "Does the body temperature change in older people?" J Clin Nurs. 2008;17(17):22847.

16. McGann KP, Marion GS, Camp L, Spangler JG. "The influence of gender and race on mean body temperature in a population of healthy older adults". Arch Fam Med. 1993;2(12):1265-7.

17. Shu-Hua Lu, Yu-Tzu Dai. "Normal body temperature and the effects of age, sex, ambient temperature and body mass index on normal oral temperature: A Prospective, Comparative study". International journal of Nursing Studies. 2009;46(5):661-8.
18. Margit L. Bleecker, Karen Bolla-Wilson, Jacqueline Agnew, Deborah A. Meyers. "Simple visual reaction time: Sex and age differences". Developmental Neuropsychology. 1987;3(2):165-172. DOI: 10.1080/87565648709540372

19. Xioa MA, Zi-Qi Yue, You-Fa Li. "The Effect of Diaphragmatic Breathing on Attention, Negative Affect and Stress in Healthy adults". Front Psychol. 2017;8:874.

20. Gomolin IH, Aung MM, Wolf-Klein G, Auerbach C. "Older is colder: temperature range and variation in older people”. J Am Geriatr Soc. 2005;53(12):2170-2.

21. Johnson JM, Kellogg DL Jr. "Thermoregulatory and thermal control in the human cutaneous circulation". Front Biosci (Schol Ed). 2010;2:825-53.

22. Ananda BB, Meena R, Madanmohan. "Immediate effect of Alternate Nostril Breathing on cardiovascular parameters and reaction time". Int Interdisciplinary Res $J$. 2014;4:297-302.

23. Shenvi D, Balasubramanian D. "A Comparative study of visual and auditory reaction times in males and females". Indian J Physiol Pharmacol. 1994;38(3):229-231.

24. Prafulla R Chandak, Jayant Makwana. "Ageing and reaction time in Indian population. People's Journal of Scientific Research". 2012;5(1):36-39.

How to cite this article: Saravanan P. L, Anu S, Kumar V. M. Gender differences on pain threshold, body temperature and reaction time among elderly population after alternate nostril breathing exercises-A comparative study. Indian J Clin Anat Physiol. 2018;5(4):483-487. 